ISSN 0103-9954

\title{
INCREMENTO, INGRESSO, MORTALIDADE EM UM REMANESCENTE DE FLORESTA OMBRÓFILA MISTA EM TRÊS BARRAS - SC
}

\author{
INCREMENT, INGROWTH, MORTALITY IN A REMNANT OF MIXED OMBROPHYLLOUS \\ FOREST IN TRÊS BARRAS, SC STATE
}

\author{
Rafael Cubas $^{1}$ Luciano Farinha Watzlawick ${ }^{2}$ Afonso Figueiredo Filho ${ }^{3}$
}

\begin{abstract}
RESUMO
A dinâmica foi estudada em um remanescente de Floresta Ombrófila Mista, localizado na Floresta Nacional de Três Barras, município de Três Barras, estado de Santa Catarina, em um período de cinco anos (20042009). Em 2004 foram instaladas e medidas 26 parcelas permanentes de 1 ha cada, sendo que as mesmas foram subdivididas em unidade de $10 \mathrm{~m} \times 50 \mathrm{~m}\left(500 \mathrm{~m}^{2}\right)$. Todas as árvores com DAP $>10 \mathrm{~cm}$ foram numeradas e mensuradas para a análise do número de espécies, gêneros, famílias botânicas, o incremento periódico anual (DAP e área basal), o ingresso e a mortalidade. Em 2009, todas as parcelas foram remedidas para avaliar as mudanças entre os dois períodos, sendo os resultados apresentados para toda a floresta (26 ha) e para as 10 espécies mais importantes. Em 2004, a floresta apresentou 721 árvores/ha passando em 2009 para 709 árvores/ha, distribuídas nas duas ocasiões, em 72 espécies, 29 famílias e 53 gêneros. Houve incremento diamétrico anual $(0,27 \mathrm{~cm} / \mathrm{ano})$ e da área basal $\left(0,28 \mathrm{~m}^{2} / \mathrm{ha} / \mathrm{ano}\right)$ para a floresta como um todo e entre as 10 espécies mais importantes, sendo Ilex theezans Mart. ex Reissek e Piptocarpha angustifolia Dusen ex Malme as espécies com menor $(0,08 \mathrm{~cm})$ e maior incremento diamétrico $(0,83 \mathrm{~cm})$, respectivamente. Considerando-se todas as espécies presentes na área de estudo, a área basal teve um acréscimo de $0,47 \mathrm{~m}^{2} / \mathrm{ha} /$ ano, indicando um contínuo crescimento em busca de atingir seu estoque completo. A taxa média anual de mortalidade foi de $2,14 \%$ e a taxa de ingresso foi de $2,01 \%$. Ilex paraguariensis A. St. Hil. apresentou a maior taxa de mortalidade $(1,86 \% /$ ano) e Cupania vernalis Cambess. a maior taxa de ingresso (2,18\%/ano). Ao serem consideradas as 10 espécies mais importantes, Ocotea porosa (Ness) Barroso apresentou maior incremento anual em diâmetro (0,43 cm/ano) e Araucaria angustifolia (Bertol.) Kuntze com maior incremento em área basal $\left(0,1874 \mathrm{~m}^{2} / \mathrm{ha} / \mathrm{ano}\right)$. De forma geral, no período de cinco anos, os resultados apontaram para pequenas alterações na dinâmica da floresta e para as 10 espécies mais importantes. Certamente, isso se deve ao estágio de sucessão avançado da floresta avaliada.
\end{abstract}

Palavras-chave: Floresta com Araucária; parcelas permanentes; dinâmica florestal.

\begin{abstract}
The dynamics was studied in a remnant of an Mixed Ombrophyllous Forest (Araucaria Moist Forest), located in Três Barras National Forest, Três Barras city, Santa Catarina state, in a period of five years (2004-2009). In 2004, it was installed and measured 26 one-hectare permanent plots, subdivided into 10 $\mathrm{m} \times 50 \mathrm{~m}\left(500 \mathrm{~m}^{2}\right)$ subplots. All trees with DBH $>10 \mathrm{~cm}$ were numbered and evaluated for the taxonomic diversity, the regular annual increment (dbh and basal area), the ingrowth and mortality. In 2009, all plots were measured again to assess changes between the two periods, and the results presented to the entire

1 Engenheiro Florestal, Msc., Doutorando em Engenharia Florestal, Centro de Ciências Rurais, Universidade Federal de Santa Maria, Av. Roraima, 1000, CEP 97105-900, Santa Maria (RS), Brasil. florestal.rafael@gmail. com

2 Engenheiro Florestal, Dr., Professor Associado do Setor de Ciências Agrárias e Ambientais, Universidade Estadual do Centro Oeste, Rua Salvador Renna c/ Padre Salvador, 875, CEP 85015-430, Guarapuava (PR), Brasil. luciano. watzlawick@pq.cnpq.br

3 Engenheiro Florestal, Dr., Professor Associado da Universidade Estadual do Centro-Oeste e Professor Sênior da Universidade Federal do Paraná, Rua Lothário Meissner, 632, CEP 80210-170, Curitiba (PR), Brasil. afigfilho@gmail.com
\end{abstract}

Recebido para publicação em 27/06/2013 e aceito em 3/12/2014

Ci. Fl., v. 26, n. 3, jul.-set., 2016 
forest (26 ha) and the 10 most important species. In 2004, the forest had 721 trees/ ha in 2009 passing for 709 trees/ha, distributed in the two occasions in 72 species, 29 families and 53 genus. There was a mean annual increment in diameter $(0.27 \mathrm{~cm} /$ year $)$, and in basal area $(0.28 \mathrm{~m} / \mathrm{ha})$ for the forest as a whole and among the 10 most important species Ilex theezans Mart. ex Reissek and Piptocarpha angustifolia Dusen ex Malme were the species with the lowest $(0.08 \mathrm{~cm})$, and highest $(0.83 \mathrm{~cm})$, diametric annual increment, respectively. Considering all species present in the study area, the basal area increased by $0.47 \mathrm{~m}^{2} / \mathrm{ha} /$ year, indicating continued growth to reach their full stock. The average annual mortality rate (AMR) was $2.14 \%$ and the annual ingrowth rate (AIR) was $2.01 \%$. Ilex paraguariensis A. St. Hil. had the highest AMR (1.86\%/year) and Cupania vernalis Cambess. the highest AIR (2.18\%/year). When considering the 10 most important species, Ocotea porosa (Ness) Barroso presented higher annual increment in diameter $(0.43 \mathrm{~cm} /$ year) and Araucaria angustifolia (Bertol.) Kuntze with higher basal area increment $\left(0.1874 \mathrm{~m}^{2} /\right.$ ha/year). Overall, in the five-year period, the results showed small changes in the dynamics of forest and for the 10 most important species. The small changes are due to the late successional stage of the forest evaluated.

Keywords: Araucaria Moist Forest; permanent plots; Forest dynamics.

\section{INTRODUÇÃO}

A Floresta Ombrófila Mista (FOM), é uma das principais unidades fitogeográficas da região sul do Brasil. Sua maior área de distribuição geográfica está associada aos locais de elevada altitude e com baixas temperaturas médias anuais, ocorrendo, de forma associada aos campos naturais, predominantemente na região do planalto meridional (HIGUCHI et al., 2012).

No estado de Santa Catarina, a Floresta Ombrófila Mista cobria originalmente 42.851,56 $\mathrm{km}^{2}(44,94 \%)$ do território, e atualmente restam $13.741,3 \mathrm{~km}^{2}$ de cobertura florestal remanescente, equivalente a $24,4 \%$ da área original (VIBRANS et al., 2013). A diminuição expressiva destes remanescentes ocorreu principalmente nos anos de 1911 a 1970, atribuída à intensa exploração madeireira das espécies com alta importância do ponto de vista econômico: Araucaria angustifolia, Ocotea porosa, Cedrela fissilis, Ocotea puberula e, através da conversão de áreas florestais para outras finalidades de uso do solo (CARVALHO, 2010).

Deste modo, há um considerável interesse em analisar o crescimento e as mudanças que ocorrem na estrutura e composição florística nos remanescentes de Floresta Ombrófila Mista, para contribuir na obtenção de informações que possam auxiliar em estratégias que visam à utilização sustentável, manutenção e conservação destes ecossistemas. Certamente, estudos detalhados sobre a dinâmica de florestas que se baseiam nos parâmetros de ingresso, crescimento e mortalidade constituem em informações que podem contribuir para a formação destas estratégias, uma vez que, esses dados fornecem importantes subsídios para a gestão sustentável de florestas naturais (SHEIL et al., 2000).

$\mathrm{O}$ ingresso refere-se às árvores que atingiram um limite mínimo de diâmetro ao longo de um determinado período de tempo, como resultado de diferentes processos, como o estabelecimento, crescimento e mortalidade de árvores (LEXERØD e EID, 2005; VANCLAY, 1994). O crescimento é definido como o aumento de dimensões de um ou mais indivíduos em uma floresta em um determinado período de tempo, o qual está influenciado pelas características da espécie interagindo com o ambiente (PRODAN et al., 1997). Tais dimensões podem ser o diâmetro, altura, área basal, volume, biomassa dentre outros (SCHAAF et al., 2005).

A mortalidade pode ser compreendida como o número de árvores que foram mensuradas inicialmente, e morreram durante um período de crescimento determinado (SANQUETTA et al., 2003). De acordo com Chao et al. (2008), as florestas compostas por espécies com baixa densidade da madeira, tendem a apresentar altas taxas de mortalidade. Para Luo e Chen (2011), a competição assimétrica é um dos principais fatores que influenciam na mortalidade.

Contudo, as estimativas desses parâmetros são obtidas, principalmente, por meio de inventário florestal contínuo com parcelas permanentes monitoradas a médio e longo prazo (FIGUEIREDO FILHO et al., 2010). Logo, é uma tarefa difícil que envolve dedicação, tendo em vista a complexidade e lentidão dos processos dinâmicos desses ecossistemas (SCHAAF et al., 2005).

De um modo geral, o ingresso, crescimento e mortalidade ocorrem em diferentes intensidades, com forte variação entre remanescentes de 
Floresta Ombrófila Mista. Estas diferenças foram observadas a partir dos estudos de Sanquetta et al. (2003), Schaaf et al. (2005), Moscovich (2006) e Figueiredo Filho et al. (2010). A variação existente nestes parâmetros é atribuída aos fatores bióticos e abióticos que envolvem os ecossistemas florestais, resultando em diferentes condições de crescimento (PICKET e CADENASSO, 2005). Portanto, isso viabiliza a realização de novos estudos de dinâmica na Floresta Ombrófila Mista para obter maior número de informações que possam contribuir no entendimento dos processos por meio dos quais ocorrem as mudanças, em níveis de espécies e para a floresta como um todo (FIGUEIREDO FILHO et al., 2010).

Diante do exposto, o presente estudo objetivou avaliar o incremento periódico anual (DAP e área basal) o ingresso e a mortalidade da floresta como um todo ( 26 ha) e das espécies mais importantes ao longo de cinco anos e ainda comparar esses resultados com demais estudos similares realizados na FOM no sul do Brasil. Dessa forma, o estudo permitirá fornecer alguns subsídios básicos à conservação e ao manejo sustentável dos remanescentes dessa tipologia florestal.

\section{MATERIAL E MÉTODOS}

Área de estudo

A área de estudo localiza-se na Floresta Nacional de Três Barras (FLONA), pertencente ao município de Três Barras, estado de Santa Catarina. A FLONA possui uma área total de 4.458,50 hectares, sendo constituída de $31,46 \%$ de florestas naturais, com predominância da vegetação denominada Floresta Ombrófila Mista (767,94 ha). Nesta formação florestal, realizou-se o presente estudo, com o uso de parcelas permanentes localizadas entre as latitudes $26^{\circ} 14^{\prime} 28,68^{\prime \prime} \mathrm{S}$ e com interseção com o meridiano $50^{\circ} 18^{\prime} 4,17^{\prime \prime}$ de longitude Oeste.

O clima da região segundo a classificação de Köppen é do tipo $\mathrm{Cfb}$, temperado, constantemente úmido, sem estação seca e com verão fresco. A precipitação média anual é de 1.588,0 mm (EPAGRI, 2001) e a temperatura média mensal mais fria é de $11,7^{\circ} \mathrm{C}$ em julho e a mais quente é de $21,3^{\circ} \mathrm{C}$, em janeiro. A altitude é de $795 \mathrm{~m}$ com relevo plano e ocorrência frequente de geadas. As associações Latossolo e Cambissolo predominam na área de estudo (MARQUES, 2007).

Registros históricos datam intensas atividades de exploração madeireira na área de estudo, realizada pela empresa "Southern Brazil Lumber \& Colonization Company" (Lumber) entre os anos de 1910 a 1940. Dentre as espécies florestais, a Araucaria angustifolia foi a mais explorada, embora, em escala bem menor, incluíam imbuia (Ocotea porosa), cedro (Cedrela fissilis) e a canelaguaicá (Ocotea puberula) (CARVALHO, 2010). O governo federal adquiriu a área que compreende a Floresta Nacional de Três Barras no ano de 1944, e a partir desta data não há registros que atestem exploração madeireira na área de estudo.

\section{Coleta dos dados}

Foram instaladas, no ano de 2004, 26 parcelas permanentes com área de um hectare cada (Figura 1). Cada unidade amostral foi dividida em 20

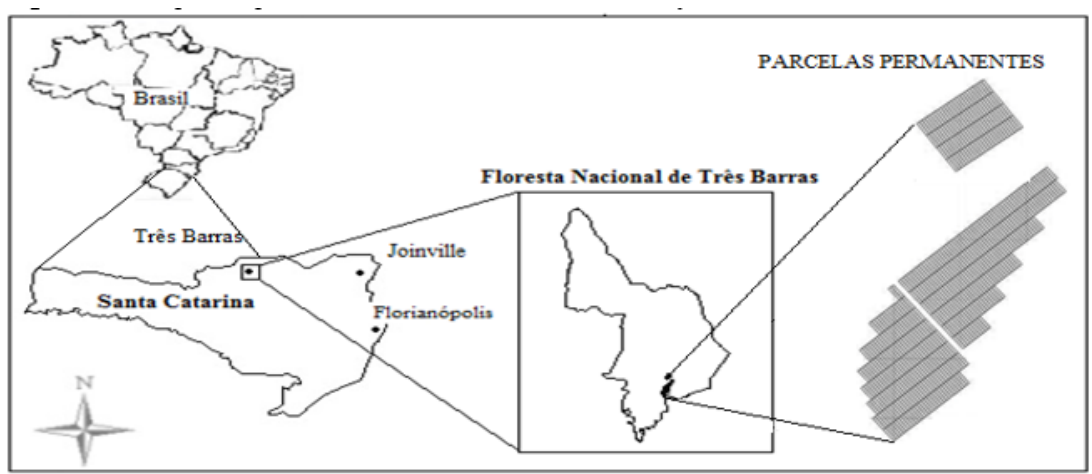

FONTE: Marques (2007), adaptado por Cubas (2011).

FIGURA 1: Localização da área de estudo e disposição das 26 parcelas permanentes (26 ha) na FLONA de Três Barras - SC.

FIGURE 1: Location map of study area and layout of 26 permanent plots (26 ha) in Três Barras National Forest, SC state. 
unidades secundárias retangulares de $500 \mathrm{~m}^{2}(10 \mathrm{mx}$ $50 \mathrm{~m}$ ), totalizando assim, 520 unidades secundárias retangulares. Todas as árvores com diâmetro a altura do peito maior ou igual a $10 \mathrm{~cm}$ foram numeradas, medidas, identificadas botanicamente e mapeadas com as coordenadas $\mathrm{X}, \mathrm{Y}$. As parcelas foram remedidas em 2009, computando-se as árvores mortas e aquelas que ingressaram.

Para análise florística da área de estudo, a identificação botânica seguiu o Sistema de Classificação APG III (Angiosperm Phylogeny Group III) (2009). A partir da identificação botânica, foram quantificadas as famílias, os gêneros e as espécies, que ocorreram na floresta nos anos de medição de 2004 e 2009.

\section{Crescimento, ingresso e mortalidade}

Com base nas medições de 2004 e 2009 foi determinado o incremento periódico anual médio em diâmetro $(I P A d)$ para a floresta e por classes de diâmetro (amplitude de $10 \mathrm{~cm}$, até o diâmetro máximo encontrado de $100 \mathrm{~cm}$ ), o incremento periódico anual em área basal por hectare $(I P A G) \mathrm{e}$ as taxas de mortalidade $(M \%)$ e ingresso (In\%).

Os incrementos foram calculados pelas fórmulas:

$$
\begin{gathered}
I P_{d}-d_{2009}-d_{2004} \\
I P A_{d}=\frac{I P_{d}}{P} \\
I P_{G}=G_{2009}-G_{2004} \\
I P A_{G}=\frac{I P_{G}}{P} \\
I n \%=\frac{N_{\text {in }}}{N_{i}} \cdot 100 \\
M \%=\frac{N_{m}}{N_{i}} \cdot 100
\end{gathered}
$$

Em que:

$\boldsymbol{P}_{d}=$ Incremento periódico em diâmetro $(\mathrm{cm})$;

$d_{2004}=$ DAP em $2004(\mathrm{~cm})$;

$d_{2009}=\mathrm{DAP}$ em $2009(\mathrm{~cm})$;

$P=$ intervalo de medição;

$\boldsymbol{P}_{G}=$ Incremento periódico em área basal $\left(\mathrm{m}^{2} / \mathrm{ha}\right) ;$

$g_{2004}=$ área basal em $2004\left(\mathrm{~m}^{2} / \mathrm{ha}\right)$;

$g_{2009}=$ área basal em $2009\left(\mathrm{~m}^{2} / \mathrm{ha}\right)$;

$N_{i n}=$ número de árvores ingressas;
$N_{i}=$ número de árvores em 2004;

$N_{m}=$ número de árvores mortas no período de 2009-2004.

Essas informações foram computadas englobando todas as espécies para a floresta como um todo e também seguindo como critério as 10 espécies de maior valor de importância (Tabela 1) (CUBAS, 2011). O processamento dos dados foi realizado com o programa Mata Nativa 2 (CIENTEC, 2001) e com o Microsoft Excel ${ }^{\circledR}$.

\section{RESULTADOS E DISCUSSÕES}

\section{Composição florística}

Em 2004 a área amostrada (26 ha) tinha 18.754 árvores, ou seja, 721 árvores por hectare. Em 2009, observaram-se poucas mudanças e foram encontradas 18.427 árvores, representadas nos anos avaliados, por 29 famílias, 53 gêneros e 72 espécies.

A florística obtida neste estudo foi similar àquela encontrada em vários trabalhos realizados em Floresta Ombrófila Mista no sul do Brasil, dentre os quais, podem-se citar: Durigan (1999), Pizatto (1999) e Gomes (2005) (Tabela 2). Contudo, Sawczuk et al. (2014), Moscovich (2006) e Ribeiro (2004) encontraram resultados superiores, que podem ser atribuídos a vários fatores, como: estágio de sucessão, grau de perturbação e características de sítio (PICKET e CADENASSO, 2005).

Os resultados revelaram ainda, que o número de árvores na floresta não representa necessariamente uma maior quantidade de famílias, gêneros e espécies, uma vez que, no presente estudo, o número de árvores foi superior ao encontrado por Sawczuk et al. (2014) e Moscovich (2006), e a riqueza foi inferior em comparação a estes dois trabalhos (Tabela 2).

Durante o período de estudo houve a predominância das famílias Myrtaceae, Lauraceae, Aquifoliaceae, Asteraceae e Fabaceae que englobaram $50 \%$ das espécies e $36 \%$ das árvores. Os resultados de outros estudos indicaram que as famílias Myrtaceae, Lauraceae e Aquifoliaceae são as mais importantes na Floresta Ombrófila Mista (Tabela 2) (SAWCZUK et al., 2014; SCHAAF et al., 2006; MOSCOVICH, 2006; BARTH FILHO, 1999). 
TABELA 1: Lista das 10 espécies com os maiores valores de importância (VI) encontradas em 26 ha de Floresta Ombrófila Mista na FLONA de Três Barras - SC.

TABLE 1: List of ten species with the highest importance value indices (VI) in 26 ha of the FLONA Ombrophyllous Mixed Forest in Três Barras, SC state.

\begin{tabular}{lcccc}
\hline \multirow{2}{*}{\multicolumn{1}{c}{ Espécie }} & DR & DoR & FR & VI \\
\cline { 2 - 5 } & $\%$ & $\%$ & $\%$ & $0-100 \%$ \\
\hline Araucaria angustifolia (Bertol.) Kuntze & 17,88 & 37,03 & 7,31 & 20,74 \\
Ocotea porosa (Ness) Barroso & 8,94 & 15,71 & 5,55 & 10,07 \\
Cupania vernalis Cambess. & 13,98 & 6,73 & 6,87 & 9,19 \\
Ocotea puberula (Rich.) Ness & 3,60 & 7,11 & 4,30 & 5,00 \\
Ilex paraguariensis A. St. Hil. & 6,13 & 1,88 & 5,62 & 4,54 \\
Syagrus romanzoffiana (Cham.) Glassmann & 4,08 & 3,88 & 4,59 & 4,18 \\
Cinnamomum vesiculosum (Ness) Kosterm & 4,08 & 3,99 & 4,10 & 4,06 \\
Jacaranda micranta Cham. & 3,76 & 1,92 & 4,58 & 3,42 \\
Clethra scabra Pers. & 3,21 & 2,45 & 2,46 & 2,71 \\
Myrcia rostrata DC. & 3,04 & 1,15 & 3,89 & 2,69 \\
\hline
\end{tabular}

Em que: $\mathrm{DR}=$ densidade relativa (\%), DoR= dominância relativa (\%), $\mathrm{FR}=$ Frequência relativa (\%) e VI= Valor de importância (\%).

TABELA 2: Resultados obtidos em trabalhos realizados na Floresta Ombrófila Mista no Sul do Brasil. TABLE 2: Results obtained from researches about Mixed Ombrophyllous Forest in Southern Brazil.

\begin{tabular}{lcccccccc}
\hline \multicolumn{1}{c}{ Variáveis } & 1 & 2 & 3 & 4 & 5 & 6 & 7 & 8 \\
\hline Densidade de plantas/ha & 708 & 567 & 440 & 586 & 611 & 677 & 855 & 852 \\
Espécies & 72 & 117 & 57 & 69 & 66 & 115 & 65 & 130 \\
Gêneros & 53 & 80 & 38 & 44 & 46 & 75 & 48 & 79 \\
Famílias & 29 & 44 & 26 & 29 & 30 & 44 & 28 & 45 \\
Diâmetro limite de inclusão (cm) & 10 & 10 & 10 & 10 & 10 & 9,5 & 9,5 & 9,5 \\
\hline \multicolumn{1}{c}{$\quad$ Área experimental (ha) } & 26 & 25 & 8 & 4 & 3,5 & 5 & 1 & 10 \\
\hline
\end{tabular}

Em que: 1. Dados desta pesquisa (Três Barras - SC); 2. Sawczuk et al. (2014) em Irati - PR; 3. Barth Filho (2002) em General Carneiro - PR; 4. Durigan (1999) em São João do Triunfo - PR; 5. Pizatto (1999) em São João do Triunfo PR; 6. Moscovich (2006) em Nova Prata - RS; 7. Gomes (2005) em São Francisco de Paula - RS; 8. Ribeiro (2004) em São Francisco de Paula - RS.

\section{Incremento em diâmetro}

$\mathrm{O}$ incremento médio anual de todas as árvores presentes nas duas medições foi de 0,27 $\mathrm{cm}$, mas variou de $0,08 \mathrm{~cm}$ para a espécie de menor incremento (Ilex theezans Mart. ex Reissek) até 0,83 cm para a espécie de maior incremento (Piptocarpha angustifolia Dusen ex Malme).

Além de Piptocarpha angustifolia, as espécies com maior incremento foram Mimosa scabrella Benth. (0,66 cm/ano), Myrcianthes cisplatensis (Cambess.) O. Berg $(0,56 \mathrm{~cm} / \mathrm{ano})$, Ocotea catharinensis Dunal $(0,51 \mathrm{~cm} / \mathrm{ano})$ e
Nectandra megapotamica Spreng. (0,44 cm/ano). Neste caso, os resultados podem ser atribuídos à baixa densidade de indivíduos destas espécies e à posição de árvores dominantes que ocupam o estrato vertical da floresta. Árvores presentes no estrato dominante apresentam maior área de copa exposta à luz, em que são transformadas pelo processo de fotossíntese em energia química (TONINI e ARCOVERDE, 2005), proporcionando maior incremento.

É importante ressaltar, que algumas espécies apresentaram incrementos negativos no período avaliado (Tabela 3). Para alguns autores (FIGUEIREDO FILHO et al., 2010; MOSCOVICH, 
2006 e SCHAAF et al., 2005), estes valores negativos podem estar associados a erros de medição ou até mesmo à diminuição no diâmetro causada tanto pela queda da casca, como pelo apodrecimento e deterioração de parte do tronco.

Dentre as 10 espécies com maior valor de importância, três espécies consideradas de alto valor econômico apresentaram incrementos expressivos: Araucaria angustifolia (Bertol.) Kuntze $(0,28 \mathrm{~cm} /$ ano); Ocotea porosa (Ness) Barroso $(0,43 \mathrm{~cm} /$ ano) e Ocotea puberula (Rich.) Ness $(0,33 \mathrm{~cm} / \mathrm{ano})$ (Tabela 3).

A Ocotea porosa apresentou um incremento superior em $40 \%$ quando comparada com as demais espécies listadas (Tabela 3). Isto se deve principalmente às taxas de incremento expressivo que esta espécie obteve em todas as classes de diâmetro, com variação de 0,33 a $0,51 \mathrm{~cm} /$ ano respectivamente nas classes de $20-30 \mathrm{~cm}$ e $40-50$ $\mathrm{cm}$. Estes resultados podem revelar a capacidade ecológica desta espécie em competir por espaço em florestas com alta densidade de árvores, uma vez que, na área de estudo foi encontrado um total de 708 árvores $/ \mathrm{ha}\left(\mathrm{G}=33,49 \mathrm{~m}^{2} / \mathrm{ha}\right)$.

Ilex paraguariensis apresentou o menor incremento $(0,09 \mathrm{~cm} / \mathrm{ano})$ no período avaliado, possivelmente, causado pela baixa vitalidade, sanidade ocasionado pelas práticas intensivas de exploração das folhas e ramos no passado (Tabela 3). Durante a coleta dos dados observaram-se sinais de podas na maioria das árvores de Ilex paraguariensis presentes no sub-bosque da floresta, podendo estar associado aos resultados de incremento obtido para esta espécie.

O incremento anual em diâmetro nas diferentes classes diamétricas para todas as espécies revelou que os maiores incrementos foram encontrados nas árvores com DAP superior a 80 $\mathrm{cm}(0,54 \mathrm{~cm} / \mathrm{ano})$ (Tabela 4). Este comportamento, entretanto, é frequentemente observado em árvores que se encontram em boas condições de crescimento, caracterizado pelos indivíduos que ocupam o estrato superior da floresta. Nestas árvores, as condições de aporte de energia solar, que afetam diretamente o crescimento, são mais homogêneas do que as condições às quais as árvores dos estratos inferiores estão sujeitas (SCHAAF et al., 2005).

Como esperado, os resultados indicaram baixo incremento nas árvores com DAP entre 10 e $20 \mathrm{~cm}(0,24 \mathrm{~cm} / \mathrm{ano})$ (Tabela 4$)$. De acordo com Schaaf et al. (2005), muitos indivíduos finos dispensam seus recursos mais na tentativa de se manterem na comunidade do que na produção de material lenhoso, podendo permanecer nessa

TABELA 3: Estatísticas do incremento periódico anual (2004-2009) em diâmetro (cm/ano) para as 10 espécies de maior valor de importância (VI) registrados em 26 ha de Floresta Ombrófila Mista na FLONA de Três Barras - SC.

TABLE 3: Statistics annual periodical increment (2004-2009) in diameter (cm/year) for the 10 species with the highest importance value (VI) recorded in 26 ha of Mixed Ombrophyllous Forest in FLONA of Três Barras, SC state.

\begin{tabular}{lcccccc}
\hline \multirow{2}{*}{ Espécie } & \multicolumn{5}{c}{ Incremento periódico anual em diâmetro (cm/ano) } \\
\cline { 2 - 7 } & $\mathrm{N} / \mathrm{ha}(2009)$ & Média & Mínimo & Máximo & Desvio padrão & $\mathrm{Cv}(\%)$ \\
\hline Araucaria angustifolia & 127 & 0,28 & $-0,22$ & 2,55 & 0,28 & 101,88 \\
Ocotea porosa & 63 & 0,43 & $-0,16$ & 1,85 & 0,311 & 72,48 \\
Cupania vernalis & 99 & 0,28 & $-0,03$ & 2,29 & 0,214 & 77,67 \\
Ocotea puberula & 26 & 0,32 & 0,00 & 1,97 & 0,295 & 92,04 \\
Ilex paraguariensis & 43 & 0,09 & $-0,19$ & 0,76 & 0,111 & 119,16 \\
Syagrus romanzoffiana & 29 & 0,27 & $-0,13$ & 2,51 & 0,348 & 127,56 \\
Cinnamomum vesiculosum & 29 & 0,34 & 0,00 & 1,62 & 0,274 & 79,63 \\
Jacaranda micranta & 27 & 0,13 & $-0,06$ & 1,69 & 0,186 & 137,37 \\
Clethra scabra & 23 & 0,27 & 0,00 & 1,59 & 0,223 & 83,3 \\
Myrcia rostrata & 22 & 0,25 & 0,00 & 1,59 & 0,212 & 83,41 \\
Demais espécies & 708 & 0,27 & 0,00 & 1,02 & 0,21 & 89,78 \\
\hline E
\end{tabular}

Em que: $\mathrm{Cv}=$ Coeficiente de variação; $\mathrm{N} / \mathrm{ha}=$ número de árvores por hectare. 
TABELA 4: Incremento periódico anual em DAP $\left(\mathrm{cm} \cdot \mathrm{ano}^{-1}\right)$, distribuídas em classes diamétricas para as 10 espécies com maior valor de importância registrado sem 26 ha de Floresta Ombrófila Mista na FLONA de Três Barras - SC.

TABLE 4: Periodic annual increment in DBH $\left(\mathrm{cm}^{-a n o^{-1}}\right)$, by diameter classes for the 10 species with the highest importance value recorded in 26 ha of Mixed Ombrophyllous Forest in FLONA of Três Barras, SC state.

\begin{tabular}{lcccccccccc}
\hline \multirow{2}{*}{\multicolumn{1}{c}{ Espécie }} & \multicolumn{10}{c}{ Classes de diâmetro (cm) } \\
\cline { 2 - 12 } & 15 & 25 & 35 & 45 & 55 & 65 & 75 & 85 & $>90$ & IPA \\
\hline Araucaria angustifolia & 0,22 & 0,27 & 0,30 & 0,34 & 0,33 & 0,25 & 0,25 & 0,58 & 0,00 & 0,28 \\
Ocotea porosa & 0,41 & 0,51 & 0,42 & 0,41 & 0,33 & 0,35 & 0,34 & 0,50 & 0,47 & 0,43 \\
Cupania vernalis & 0,27 & 0,35 & 0,20 & 0,12 & 0,23 & 0,19 & & - & - & 0,28 \\
Ocotea puberula & 0,23 & 0,27 & 0,35 & 0,44 & 0,47 & 0,52 & - & - & - & 0,33 \\
Ilex paraguariensis & 0,09 & 0,11 & - & - & - & - & - & - & - & 0,09 \\
Syagrus romanzoffiana & 0,48 & 0,19 & 0,07 & - & - & - & - & - & - & 0,26 \\
Cinnamomum vesiculosumyyyyyyyyyyyyyyyyy & 0,31 & 0,37 & 0,45 & 0,47 & 0,34 & 0,06 & - & - & - & 0,35 \\
Jacaranda micranta & 0,12 & 0,21 & 0,14 & 0,25 & - & - & - & - & - & 0,14 \\
Clethra scabra & 0,23 & 0,32 & 0,40 & 0,18 & - & - & - & - & - & 0,28 \\
Myrcia rostrata & 0,25 & 0,32 & 0,25 & 0,06 & - & - & - & - & - & 0,26 \\
\hline Demais espécies: & 0,24 & 0,31 & 0,32 & 0,36 & 0,35 & 0,28 & 0,28 & 0,54 & 0,36 & 0,27 \\
\hline
\end{tabular}

condição por anos.

Quando analisadas as árvores com diâmetro entre 20 a $30 \mathrm{~cm}$, observaram-se valores de incremento periódico anual $(0,31 \mathrm{~cm} / \mathrm{ano})$ similar às árvores com diâmetro entre 40 a $50 \mathrm{~cm}(0,36 \mathrm{~cm} /$ ano), atribuído certamente, pelo incremento superior que as árvores de Ocotea porosa apresentaram nas menores classes de diâmetro $(0,51 \mathrm{~cm} / \mathrm{ano})$ (Tabela 4).

Dentre as espécies de maior valor de importância, Araucaria angustifolia apresentou incremento similar, com sete das dez espécies analisadas. Este resultado pode ser atribuído ao incremento expressivo que esta espécie obteve nas árvores que compreendem os centros de classes: $35,45,55$ e $85 \mathrm{~cm}$ (Tabela 4), indicando que, a Araucaria angustifolia tende a permanecer como uma das espécies mais importantes na floresta avaliada.

Outro aspecto relevante que deve ser ressaltado é que estes resultados também revelaram uma tendência de Syagrus romanzoffiana evoluir para as melhores posições de importância na floresta, uma vez que, as árvores entre 10 a 20 $\mathrm{cm}$, apresentaram os valores mais elevados de incremento $(0,48 \mathrm{~cm} / \mathrm{ano})$, superando até mesmo, a Ocotea porosa na menor classe de diâmetro $(0,41$ $\mathrm{cm} / \mathrm{ano}$ ) (Tabela 4).
O diâmetro médio foi muito similar em nove de 10 estudos que avaliaram a dinâmica da Floresta Ombrófila Mista no sul do Brasil (Tabela 5). Além disso, o incremento diamétrico anual encontrado neste estudo $(0,27 \mathrm{~cm} / \mathrm{ano})$, foi próximo ao obtido por Figueiredo Filho et al. (2010), Rossi (2007) e Schaaf et al. (2005). Por fim, o incremento médio anual encontrado neste estudo foi mais que o dobro ao encontrado por Barth Filho (2002) (Tabela 5).

\section{Incremento em área basal}

A área basal por hectare aumentou de 31,14 $\mathrm{m}^{2} /$ ha em 2004 para $33,49 \mathrm{~m}^{2} / \mathrm{ha}$ em 2009. Em nível de incremento das espécies mais importantes, destacaram-se de forma expressiva a Araucaria angustifolia $\left(0,187 \mathrm{~m}^{2} / \mathrm{ha} / \mathrm{ano}\right)$, seguida pela Ocotea porosa $\left(0,126 \mathrm{~m}^{2} / \mathrm{ha} / \mathrm{ano}\right)$ (Tabela 6$)$. Somadas, estas duas espécies representaram 53\% da área basal total encontrada $\left(33,49 \mathrm{~m}^{2} / \mathrm{ha}\right)$ no ano de 2009 , o que demonstra a importância destas espécies na estrutura do remanescente de Floresta Ombrófila Mista avaliado.

A área basal por hectare encontrada no presente estudo foi similar a vários outros trabalhos, como Figueiredo Filho et al. (2010), Pizzato (1999), Durigan (1999) e Rossi (2007). Contudo, Gomes (2005) e Ribeiro (2004) obtiveram resultados 
TABELA 5: Incremento anual médio em diâmetro e diâmetro médio encontrado por vários autores que trabalharam com Floresta Ombrófila Mista no sul do Brasil.

TABLE 5: Mean annual increment in diameter and average diameter found by several authors who have worked with Mixed Ombrophyllous Forest in southern Brazil.

\begin{tabular}{lccccccccc}
\hline \multicolumn{1}{c}{ Variáveis } & 1 & 2 & 3 & 4 & 5 & 6 & 7 & 8 & 9 \\
\hline Área amostrada (ha) & 26,00 & 25,00 & 8,00 & 4,00 & 3,50 & 5,00 & 1,00 & 10,00 & 3,50 \\
Diâmetro limite de inclusão (cm) & 10,00 & 10,00 & 10,00 & 10,00 & 10,00 & 9,50 & 9,50 & 9,50 & 10,0 \\
Diâmetro médio (cm) & 21,18 & 24,30 & 24,60 & 23,80 & 21,80 & 22,10 & 21,45 & 21,95 & 21,00 \\
Incremento diamétrico (cm/ano) & 0,27 & 0,24 & 0,13 & 0,34 & 0,18 & 0,17 & - & - & 0,25 \\
\hline
\end{tabular}

Em que: 1. Dados desta pesquisa (Três Barras - SC); 2. Figueiredo Filho et al. (2010) em Irati - PR; 3. Barth Filho (2002) em General Carneiro - PR; 4. Durigan (1999) em São João do Triunfo - PR; 5. Pizzatto (1999) em São João do Triunfo - PR; 6. Moscovich (2006) em Nova Prata - RS; 7. Gomes (2005) em São Francisco de Paula - RS; 8. Ribeiro (2004) em São Francisco de Paula - RS; 9. Rossi (2007) em São João do Triunfo - PR.

TABELA 6: Incremento periódico médio anual em área basal (2004-2009) para todas as espécies da área amostrada (26 ha) e para as 10 espécies de maior valor de importância na Floresta Ombrófila Mista da FLONA de Três Barras - SC.

TABLE 6: Average annual periodical increment in basal area (2004-2009) for all species of the sampled area (26 ha) and for the 10 species with the highest importance value in the Mixed Ombrophyllous Forest FLONA of Três Barras, SC state.

\begin{tabular}{|c|c|c|c|c|c|}
\hline \multirow{3}{*}{ Nome científico } & \multirow{2}{*}{ N/ha } & \multirow{2}{*}{ G/ha } & \multirow{2}{*}{$\mathrm{N} / \mathrm{ha}$} & \multirow{2}{*}{ G/ha } & \multirow{2}{*}{$\frac{\text { Incremento em área basal }}{2004-2009}$} \\
\hline & & & & & \\
\hline & 2004 & & 2009 & & $\mathrm{~m}^{2} / \mathrm{ha} /$ ano \\
\hline Araucaria angustifolia & 124 & 11,465 & 127 & 12,402 & 0,1874 \\
\hline Ocotea porosa & 61 & 4,632 & 67 & 5,263 & 0,1262 \\
\hline Cupania vernalis & 95 & 1,946 & 102 & 2,254 & 0,0616 \\
\hline Ocotea puberula & 31 & 2,462 & 26 & 2,382 & $-0,0160$ \\
\hline Ilex paraguariensis & 49 & 0,711 & 44 & 0,628 & $-0,0166$ \\
\hline Syagrus romanzoffiana & 28 & 1,151 & 29 & 1,298 & 0,0294 \\
\hline Cinnamomum vesiculosum & 30 & 1,181 & 30 & 1,337 & 0,0312 \\
\hline Jacaranda micrantha & 27 & 0,599 & 27 & 0,641 & 0,0084 \\
\hline Clethra scabra & 27 & 0,824 & 23 & 0,821 & $-0,0006$ \\
\hline Myrcia rostrata & 22 & 0,338 & 22 & 0,384 & 0,0092 \\
\hline Média - Todas as espécies & 730 & 31,140 & 717 & 33,49 & 0,2790 \\
\hline
\end{tabular}

superiores, atribuídos certamente pela alta densidade de árvores nas áreas de estudo ( 855 e 852 , respectivamente) (Tabela 7). Portanto, os valores de área basal obtidos neste estudo se assemelham à maioria dos remanescentes de Floresta Ombrófila Mista do sul do Brasil.

Ao serem comparados os resultados dos incrementos periódicos em área basal para a floresta, obtidos neste trabalho, observou-se que o valor foi próximo ao obtido por Figueiredo Filho et al. (2010) e Schaaf et al. (2005) e inferior aos demais estudos (Tabela 7). Esses resultados podem estar relacionados com as diferenças entre estágios sucessionais, histórico de perturbações/intervenções (exploração madeireira) que a floresta teve no passado, além da área amostrada, limite de inclusão, período de monitoramento (FIGUEIREDO FILHO et al., 2010) e qualidade de sítio.

\section{Mortalidade e ingresso}

$\mathrm{Na}$ área amostrada (26 ha) ocorreu uma 
TABELA 7: Área basal e incremento periódico encontrados em alguns estudos realizados em Floresta Ombrófila Mista no sul do Brasil.

TABLE 7: Basal area and periodic increment found in several studies in Ombrophyllous Mixed Forest in southern Brazil.

\begin{tabular}{lcccccccccc}
\hline \multirow{2}{*}{\multicolumn{1}{c}{ Variáveis }} & \multicolumn{10}{c}{ Autores } \\
\cline { 2 - 11 } & 1 & 2 & 3 & 4 & 5 & 6 & 7 & 8 & 9 & 10 \\
\hline $\mathrm{G}\left(\mathrm{m}^{2} / \mathrm{ha}\right)$ & 33,49 & 30,10 & 25,70 & 28,50 & 37,00 & 33,07 & 33,80 & 45,10 & 46,00 & 35,00 \\
$\begin{array}{l}\text { Incremento em } \mathrm{G}\left(\mathrm{m}^{2} /\right. \\
\text { ha/ano) }\end{array}$ & 0,28 & 0,23 & - & 0,24 & 0,71 & 0,35 & 0,37 & - & - & 0,50 \\
$\mathrm{~N} /$ ha & 708,00 & 567,00 & 440,00 & 244,00 & 586,00 & 611,00 & 677,00 & 855,00 & 852,00 & 669,00 \\
$\begin{array}{l}\text { Diâmetro limite } \\
\text { inclusão (cm) }\end{array}$ & 10,00 & 10,00 & 10,00 & 20,00 & 10,00 & 10,00 & 9,50 & 9,50 & 9,50 & 10,00 \\
\hline Área amostrada (ha) & 26,00 & 25,00 & 8,00 & 9,00 & 4,00 & 3,50 & 5,00 & 10,00 & 10,00 & 3,50 \\
\hline
\end{tabular}

Em que: 1. Dados desta pesquisa (Três Barras - SC); 2. Figueiredo Filho et al. (2010) em Irati - PR; 3. Barth Filho (2002) em General Carneiro - PR; 4.Schaaf et al. (2005) em São João do Triunfo - PR; 5. Durigan (1999) em São João do Triunfo - PR; 6. Pizatto (1999) em São João do Triunfo, PR; 7. Moscovich (2006) em Nova Prata - RS; 8. Gomes (2005) em São Francisco de Paula - RS; 9. Ribeiro (2004) em São Francisco de Paula - RS; 10. Rossi (2007) em São João do Triunfo - PR.

mortalidade de 1.568 árvores, ou seja, 60 árvores/ha ano e um ingresso de 1.241 árvores ou 47 árvores/ ha no período de cinco anos. Isto indica que houve um balanço negativo no número de indivíduos no período de remedição, resultando em uma perda líquida de 12,57 árvores/ha, com uma taxa média de mortalidade anual de $2,14 \%$, superior à taxa de ingresso, que foi de 1,32\% (Tabelas 8).

Este resultado pode ser atribuído principalmente à mortalidade das árvores com os menores diâmetros (10 a $20 \mathrm{~cm}$ ), que representaram $80,54 \%$ do total das árvores que saíram do sistema. Sem ter espaço suficiente para crescer, estas árvores pequenas, cuja capacidade de competir por recursos é menor, morrem em virtude do aumento da competição e da supressão, conforme as populações florestais se aproximam do número limite de árvores de certo tamanho, que possam coexistir em determinada área (MEYER et al., 2013).

Dentre as espécies de maior valor de importância, Ilex paraguariensis apresentou a maior taxa de mortalidade (4,20\%). Esses resultados estão associados à baixa vitalidade e sanidade das árvores desta espécie presentes no sub-bosque da floresta e das práticas de exploração de folhas e ramos no passado, conforme foi mencionado anteriormente. É importante destacar, que no período avaliado de cinco anos, Ilex paraguariensis permaneceu na mesma posição de importância na floresta $\left(5^{\circ}\right.$ posição), com taxa de ingresso anual de 1,86\% (Tabela 8).
A mortalidade expressiva de árvores de Ilex paraguariensis também foi observada em estudo conduzido por Sawczuk et al. (2014) em Floresta Ombrófila Mista, em que atribuíram estes resultados a uma perda natural da posição de importância desta espécie na floresta amostrada.

Quando se analisa o número de indivíduos ingressos, observou-se as maiores taxas de ingresso para a Cupania vernalis (1,35 \%/ano) em comparação às demais, devido certamente às características ecológicas da espécie e às condições favoráveis de sítio (Tabela 8).

No entanto, Araucaria angustifolia apresentou baixas taxas de ingresso e mortalidade, indicando que os indivíduos de pequeno porte pouco se desenvolveram no sub-bosque, aguardando, a maior disponibilidade de luz causada pela abertura do dossel. Isto indica que as árvores com os menores diâmetros desta espécie apresentam grande capacidade de resistência à forte concorrência no estrato inferior da floresta (SOLIGO, 2009).

O presente estudo demonstrou que a taxa anual média de mortalidade, foi superior à maioria dos outros locais da Floresta Ombrófila Mista no sul do Brasil. Entretanto, Durigan (1999) obteve resultado inferior, possivelmente atribuído ao pequeno período de monitoramento (1997-1998) (Tabela 9).

A mortalidade em florestas naturais pode ser influenciada pela presença de diferentes fatores, como: composição das espécies, grau de 
TABELA 8: Ingressos de árvores das 10 espécies de maior valor de importância e para todas as espécies encontradas em 26 ha de Floresta Ombrófila Mista na FLONA de Três Barras - SC.

TABLE 8: Tree ingrowth of the 10 species of greatest importance value and for all species studied in the area found in 26 ha of Ombrophyllous Mixed Forest in FLONA of Três Barras, SC state.

\begin{tabular}{|c|c|c|c|c|c|c|c|}
\hline \multirow{2}{*}{ Espécie } & \multirow{2}{*}{ N/ha (2004) } & \multicolumn{2}{|c|}{ Ingresso/c. classe } & \multirow{2}{*}{ Total/ingresso } & \multirow{2}{*}{ In $(\%)$} & \multirow{2}{*}{$\mathrm{M} / \mathrm{ha}$} & \multirow{2}{*}{ T.A.M (\%) } \\
\hline & & $15,0 \mathrm{~cm}$ & $25,0 \mathrm{~cm}$ & & & & \\
\hline Araucaria angustifolia & 124 & 77 & 2 & 79 & 0,49 & 0,69 & 0,11 \\
\hline Ocotea porosa & 61 & 72 & 0 & 72 & 0,91 & 0,12 & 0,04 \\
\hline Cupania vernalis & 95 & 267 & 2 & 269 & 2,17 & 6,42 & 1,35 \\
\hline Ocotea puberula & 31 & 3 & 0 & 3 & 0,08 & 5,23 & 3,42 \\
\hline Ilex paraguariensis & 49 & 119 & 0 & 119 & 1,86 & 10,35 & 4,2 \\
\hline Syagrus romanzoffiana & 28 & 35 & 5 & 40 & 1,12 & 0,15 & 0,11 \\
\hline Cinnamomum vesiculosum & 30 & 23 & 2 & 25 & 0,64 & 2,08 & 1,38 \\
\hline Jacaranda micrantha & 27 & 22 & 0 & 22 & 0,63 & 0,96 & 0,72 \\
\hline Clethra scabra & 27 & 9 & 0 & 9 & 0,26 & 4,58 & 3,4 \\
\hline Myrcia rostrata & 22 & 60 & 0 & 60 & 2,13 & 2,35 & 2,17 \\
\hline Demais espécies: & 721,31 & 1220 & 15 & 1235 & 1,32 & 60,31 & 2,14 \\
\hline
\end{tabular}

Em que: $\mathrm{M} / \mathrm{ha}=$ Mortalidade por hectare; In = Taxa anual de ingresso; TAM = Taxa anual de mortalidade.

competição, ocorrência de distúrbios naturais, disponibilidade de luz, características de sítio e período de monitoramento (FRELICH, 2002; CHAO et al., 2008; LUO e CHEN, 2011; DUCHESNE e PRÉVOST, 2013). Estes mesmos fatores podem estar associados à variabilidade entre os resultados listados na Tabela 9.

Com relação à taxa média anual de ingresso, este estudo demonstrou resultados semelhantes a muitos estudos nas FOM do sul do Brasil (FIGUEIREDO FILHO et al., 2010; SCHAAF et al., 2006; DURIGAN, 1999) (Tabela 9).

\section{CONCLUSÕES}

As famílias Myrtaceae, Lauraceae e Aquifoliaceae foram as mais importantes em número de espécies na área de estudo, sendo similar a outras FOM do sul do Brasil.

A Floresta Ombrófila Mista apresenta grande variabilidade nos parâmetros de ingresso, crescimento e mortalidade.

O incremento médio anual em diâmetro apresentou grande variabilidade entre as espécies mais importantes avaliadas, com variação de 0,09 $\mathrm{cm} /$ ano a $0,43 \mathrm{~cm} /$ ano, e de $0,27 \mathrm{~cm} /$ ano quando considerada a floresta como um todo.

Algumas espécies apresentaram crescimento nulo no período e outras tiveram grandes incrementos médios anuais de até $0,83 \mathrm{~cm}$.

A floresta continua crescendo em área basal indicando claramente que ainda está evoluindo em busca de atingir seu estoque completo, ainda que a mortalidade tenha sido maior que o ingresso. Isto indica que as árvores longevas e de dossel estão migrando para classes de diâmetro maiores, aumentando as dificuldades de sobrevivência para as árvores dos extratos inferiores.

Araucaria angustifolia e Ocotea porosa são as espécies mais expressivas da floresta em quantidade de indivíduos e em termos de área basal, apresentando um grau de ocupação na floresta de $53 \%$, tendo aumentado sua dominância nos cinco anos de monitoramento em $1 \%$.

Árvores nas classes de diâmetro inferiores apresentaram as menores taxas de crescimento em diâmetro e maiores mortalidades, fato esperado pelo alto grau de competição.

\section{REFERÊNCIAS BIBLIOGRÁFICAS}

APG III. Angiosperm Phylogeny Group. An update of the Angiosperm Phylogeny Group classification for the orders and families of flowering plants. Botanical Journal of the Linnean Society, London, v. 161, p. 105-121, 2009.

BARTH FILHO, N. F. Delineamentos de um sistema de monitoramento de crescimento e 
TABELA 9: Taxa anual de mortalidade e ingresso pesquisados em Floresta Ombrófila Mista por alguns autores no sul do Brasil.

TABLE 9: Annual mortality and ingrowth from researches with Ombrophyllous Mixed Forest by several authors in southern Brazil.

\begin{tabular}{lcccccccc}
\hline \multirow{2}{*}{\multicolumn{1}{c}{ Variáveis }} & \multicolumn{8}{c}{ Autores } \\
\cline { 2 - 9 } & 1 & 2 & 3 & 4 & 5 & 6 & 7 & 8 \\
\hline DAP limite de inclusão (cm) & 10,00 & 10,00 & 10,00 & 20,00 & 10,00 & 3,50 & 5,00 & 3,50 \\
Taxa de mortalidade anual (\%) & 2,14 & 1,77 & 1,84 & 1,15 & 0,21 & 1,49 & 7,40 & 1,10 \\
Taxa de ingresso anual (\%) & 2,01 & 1,38 & 5,03 & 1,32 & 1,62 & 3,50 & 3,05 & 3,00 \\
\hline
\end{tabular}

Em que: 1. Dados desta pesquisa (Três Barras - SC); 2. Figueiredo Filho et al. (2010) em Irati - PR; 3. Barth Filho (2002) em General Carneiro - PR; 4. Schaaf et al. (2006) em São João do Triunfo - PR; 5. Durigan (1999) em São João do Triunfo - PR; 6. Pizatto (1999) em São João do Triunfo - PR; 7. Moscovich (2006) em Nova Prata - RS; 8. Rossi (2007) em São João do Triunfo - PR.

produção em campo para florestas naturais: aplicação na floresta com araucária. Curitiba, 2002. 86 f. Dissertação (Mestrado em Engenharia Florestal) - Universidade Federal do Paraná, Curitiba, 2002.

CARVALHO, M. M. X de. Uma grande empresa em meio à floresta: a história da devastação da floresta com araucária e a Souther Brazil Lumber and colonization (1870-1970). 2010, 313p. Tese (Doutor em história) - Universidade Federal de Santa Catarina, Florianópolis, 2010.

CHAGAS, R. K. et al. Crescimento diametral de espécies arbóreas em Floresta Estacional Semidecidual ao longo de seis anos. In: DURIGAN, G.; VILAS-BOAS, O. Pesquisas em conservação e recuperação ambiental no oeste paulista. São Paulo: Páginas; Letras, p 265-290, 2004.

CHAO, K. J; et al. Growth and wood density predict tree mortality in Amazon forests. Journal of Ecology. v. 96, p. 281-292, 2008.

CIENTEC. S. A. Consultoria e Desenvolvimento de sistemas. Mata nativa - Sistema para análise fitossociológica e elaboração de planos de manejo de florestas nativas. Viçosa, MG: 2001. CD-ROM. CUBAS, R. Florística, estrutura e dinâmica em uma Floresta Ombrófila Mista no norte do estado de Santa Catarina. 2011. 133 f. Dissertação (Mestrado em Ciências Florestais) - Universidade Estadual do Centro Oeste, Irati, 2011.

DUCHESNE, L.; PRÉVOST, M. Canopy disturbance and inter tree competition: implications for tree growth and recruitment in two yellow birch-conifer stands in Quebec, Canada. Canadian Journal of Forest Research. v. 18, p. 168-178, 2013.

DURIGAN, M. E. Florística, dinâmica e análise proteica de uma Floresta Ombrófila Mista em São João do Triunfo, PR. 1999. 125 f. Dissertação (Mestrado em Engenharia Florestal) - Universidade Federal do Paraná, Curitiba, 1999.

EPAGRI. Dados e informações biofísicas da Unidade de planejamento Regional Planalto Norte Catarinense - UPR 4. Ed. EPAGRI. Florianópolis, 2001.

FRELICH, L. E. Forest Dynamics and disturbance regimes: Studies from temperate Evergreen Deciduous Forests. Cambridge University Press, London. 2002. 266 p.

FIGUEIREDO FILHO, A. et al. Crescimento, mortalidade, ingresso e distribuição diamétrica em Floresta Ombrófila Mista. Floresta, Curitiba, v. 4, n. 4, p. 763-776, 2010.

GOMES, J. F. Classificação e crescimento de grupos ecológicos na Floresta Ombrófila Mista da FLONA de São Francisco de Paula, RS. Santa Maria, 2005. 75 f. Dissertação (Mestrado em Ciências Florestais) - Universidade Federal de Santa Maria, Curitiba, 2005.

HIGUSHI, et al. Influência de variáveis ambientais sobre o padrão estrutural e florístico do componente arbóreo, em um fragmento de Floresta Ombrófila Mista Montana em Lages, SC. Ciência florestal., Santa Maria, v.22 , n.1 , p. 79-90 , 2012.

LEXERØ, D.; EID, T. Recruitment Models for Norway Spruce, Scots Pine, Birch and Other Broad leaves in Young Growth Forests in Norway. Silva Fennica, v. 39, n. 3. p. 391 - 406. 2005.

LUO, Y.; CHEN, Y. H. Competition, species interaction and ageing control tree mortality in boreal forests. Journal of Ecology. V. 99, p. 1470-1480, 2011.

MARQUES, A. C. Planejamento da Paisagem 
da Floresta Nacional de Três Barras (Três Barras - SC): subsídios ao plano de manejo. 2007. 145 f. Dissertação (Mestrado em Geografia) - Universidade Federal do Paraná, Curitiba, 2007. MEYER, E. A. et al. Ajuste do modelo de Reineke para estimativa da linha de máxima densidade na Floresta Estacional Decidual no Rio Grande do Sul. Revista Árvore. v. 37, n.4, p. 669-678, 2013. MOSCOVICH, F. A. Dinâmica de crescimento de uma Floresta Ombrófila Mista em Nova Prata, RS. Santa Maria, 2006. 130 f. Tese (Doutorado em Ciências Florestais) - Programa de Pós-Graduação em Engenharia Florestal, Universidade Federal de Santa Maria, 2006.

PRODAN, M. et al. Mensura Forestal. San Jose, C.R.: Deutsche Gesellschaft fur Technische Zusammenarbeit (GTZ) GmbH: Institute Interamericano de Cooperación para la Agricultura (IICA), 1997, $561 \mathrm{p}$.

PICKETT, S. T. A.; CADENASSO, M. L. Vegetation dynamics in: van der Maarel, E. (Ed.) Vegetation Ecology. Black well Publishing, Oxford, UK, p. 172-198, 2005.

PIZZATTO, W. Avaliação biométrica da estrutura e da dinâmica de uma Floresta Ombrófila Mista em São João do Triunfo, PR: 1995 a 1998. Curitiba, 1999. 172 f. Dissertação (Mestrado em Engenharia Florestal) - Universidade Federal do Paraná, Curitiba, 1999.

RIBEIRO, S. B. Classificação e ordenação da comunidade arbórea da Floresta Ombrófila Mista da FLONA de São Francisco de Paula, RS. Santa Maria, 2004. 181 f. Tese (Doutorado em Ciências Florestais) - Universidade Federal de Santa Maria, Santa Maria, 2004.

ROSSI, L. M. B. Processo de difusão para simulação da dinâmica de Floresta Natural. Curitiba, 2007. 168 f. Tese (Doutorado em Engenharia Florestal) - Universidade Federal do Paraná, Curitiba, 2007.
SANQUETTA, C. R. et al. Crescimento, mortalidade e recrutamento em duas florestas de Araucária (Araucaria angustifolia) no estado do Paraná, Brasil. Revista Ciências Exatas e Naturais, v. 5, n. 1, p. 101-112, 2003.

SAWCZUK, A. et al. Alterações na estrutura horizontal, no período de 2002-2008, em Floresta Ombrófila Mista no Centro-Sul do estado do Paraná. Ciência florestal, v. 24, n. 1, p. 149-160, 2014.

SOLIGO, A. J. Crescimento da Araucaria angustifolia regenerada sob Pinus elliottii e em povoamento homogêneo interplantado com Pinus spp. Santa Maria, 2009. 132 f. Dissertação (Mestrado em engenharia florestal) - Universidade Federal de Santa Maria, Santa Maria, 2009.

SCHAAF, L. B. et al. Incremento diamétrico e em área basal no período de 1979-2000 de espécies arbóreas de uma Floresta Ombrófila Mista localizada no sul do Paraná. Revista Floresta, Curitiba, v.35, n.2, p.271-290, 2005.

SCHAAF, L. B. et al. Alteração na estrutura diamétrica de uma Floresta Ombrófila Mista no período entre 1979 e 2000 . Revista árvore, Viçosa, v. 30, n. 2, p. 283-295, 2006.

SHEIL, D.; JENNINGS, S.; SAVILL, P. Long-term permanent plot observations of vegetation dynamics in Budongo, a Ugandan rain forest. Journal of Tropical Ecology, v. 16, p. 765-800, 2000.

TONINI, H.; ARCO-VERDE, F. Morfologia da copa para avaliar o espaço vital de quatro espécies nativas da Amazônia. Pesquisa Agropecuária brasileira, Brasília, v. 40, n. 7, p. 633-638, 2005.

VIBRANS, A. C. et al. Floresta Ombrófila Mista: Inventário Florístico Florestal de Santa Catarina, V.3. 1. ed. Blumenau: Edifurb, 2013. $440 \mathrm{f}$.

VANCLAY, J. K. Modelling Forest Growth and Yield: Applications to Mixed Tropical Forest. Walling ford: CAB INTERNATIONAL, 1994, $312 \mathrm{p}$. 